\title{
POTENCIAL DE PRODUÇÃO DE BIOGÁS REMANESCENTE NOS EFLUENTES DE BIODIGESTORES ABASTECIDOS COM DEJETOS DE SUÍNOS, COM E SEM SEPARAÇÃO DA FRAÇÃO SÓLIDA, E CONDUZIDOS SOB DIFERENTES TEMPOS DE RETENÇÃO HIDRÁULICA
}

\author{
MARCO A. P. ORRICO JÚNIOR ${ }^{1}$, ANA C. A. ORRICO ${ }^{2}$, JORGE DE LUCAS JÚNIOR ${ }^{3}$
}

RESUMO: O objetivo foi avaliar o potencial poluidor remanescente dos efluentes de biodigestores abastecidos com dejetos de suínos com separação da fração sólida (CSFS) e sem separação da fração sólida (SSFS), e conduzidos sob diferentes tempos de retenção hidráulica (TRH). Os efluentes utilizados eram de biodigestores semicontínuos manejados com TRH de 15; 22; 29 e 36 dias, com e sem separação da fração sólida. Foram utilizados biodigestores batelada, que permaneceram em operação por todo o tempo em que houve produção de biogás (60 dias). Foram avaliadas a produção e a qualidade do biogás, bem como os potenciais de produção por $\mathrm{kg}$ de sólidos totais e sólidos voláteis, e as demandas química e bioquímica de oxigênio. Utilizou-se do delineamento inteiramente casualisado, em esquema fatorial $2 \times 4$, com três repetições por tratamento. Foram encontrados potenciais de produção de 385 e 117 litros de $\mathrm{CH}_{4} \mathrm{~kg}^{-1}$ de SV adicionados no material SSFS e CSFS, respectivamente, no menor TRH (15 dias), e potenciais de produção de 74 e 18 litros de $\mathrm{CH}_{4} \mathrm{~kg}^{-1}$ de $\mathrm{SV}$ adicionados no material SSFS e CSFS, respectivamente, no maior TRH (36 dias).

PALAVRAS-CHAVE: metano, sólidos totais, biodigestão anaeróbia.

\section{PRODUCTION POTENTIAL OF BIOGAS REMAINING ON EFFLUENTS FROM BIODIGESTERS THAT OPERATE WITH SWINE MANURE, WITH AND WITHOUT SOLID FRACTIONS SEPARATION UNDER DIFFERENT HYDRAULIC RETENTION TIMES}

\begin{abstract}
The objective of this work was to evaluate the polluting potential from the remainings of effluents from biodigesters that operate with swine manure with the separation of the solid fraction and without the separation of the solid fraction, both under different hydraulic retention times (HRT). For the biodigestion trial, the effluents from semi-continuous biodigesters were processed with $15 ; 22 ; 29$ and 36 days of hydraulic retention, with and without the separation of the solid fraction. In this part of the work batch biodigesters were used, which were kept in the operation as long as biogas was produced (60 days). It was evaluated: biogas production and quality and yield potential, the potential production per $\mathrm{kg}$ of total solids and volatile solids and chemical and biochemical demands for oxygen. Production potential found with separation of the solid fraction was 385 liter of $\mathrm{CH}_{4} \mathrm{~kg}^{-1}$ of VS added and the effluent without the separation of the solid fraction was 117 liter of $\mathrm{CH}_{4} \mathrm{~kg}^{-1}$ of VS added, for the shorter HRT (15 days) and production potentials of 74 and 18 liters of $\mathrm{CH}_{4} \mathrm{~kg}^{-1}$ of VS added to the substrates with swine manure with the separation of the solid fraction and without the separation of the solid fraction respectively for the longer HRT (36 days).
\end{abstract}

KEYWORDS: methane, total solid, anaerobic biodigestion.

\section{INTRODUÇÃO}

Um problema que vem recebendo atenção especial nos últimos anos, não apenas no que se refere à suinocultura, mas à produção animal em geral, é o alto potencial de emissão de gases de efeito estufa provenientes da degradação dos dejetos em lagoas de estabilização. Esses gases impedem a saída da radiação solar que é refletida na superfície da Terra para o espaço, contribuindo, assim, para o aumento da temperatura global (UNFCCC, 2006).

\footnotetext{
${ }^{1}$ Doutorando em Zootecnia, Faculdade de Ciências Agrárias e Veterinárias - UNESP, Jaboticabal - SP, marcoorrico@ yahoo.com.br

${ }^{2}$ Profa. Adjunta, Faculdade de Ciências Agrárias da Universidade Federal da Grande Dourados, anaorrico@ufgd.edu.br

${ }^{3}$ Prof. Titular, Faculdade de Ciências Agrárias e Veterinárias - UNESP, Jaboticabal - SP, jlucas @ fcav.unesp.br

Recebido pelo Conselho Editorial em: 19-3-2008
}

Aprovado pelo Conselho editorial em: 23-10-2009

Eng. Agríc., Jaboticabal, v.29, n.4, p.679-686, out./dez. 2009 
Esses gases trazem grande preocupação devido ao seu tempo de vida na atmosfera e seu potencial de aquecimento global com relação ao $\mathrm{CO}_{2}\left(\right.$ o $\mathrm{CO}_{2}$ é o principal gás do efeito estufa, por isso foi eleito como sendo índice 1 para o aquecimento global, e os demais gases são comparados a ele). Segundo a UNFCCC (2006), a vida média desses gases na atmosfera seria de 12 anos para o $\mathrm{CH}_{4}$ e 120 anos para o $\mathrm{N}_{2} \mathrm{O}$ e os respectivos potenciais de aquecimento global iguais a 21 e 310 , ou seja, 21 e 310 vezes mais perigoso que o $\mathrm{CO}_{2}$.

A única forma de evitar a emissão desses gases seria a captação e posterior combustão, em que o $\mathrm{CH}_{4}$ e $\mathrm{N}_{2} \mathrm{O}$ seriam transformados em $\mathrm{CO}_{2}$ e $\mathrm{N}_{2}$ após a queima, reduzindo, assim, a contribuição para o aquecimento global. $\mathrm{O}$ uso de biodigestores é uma forma segura de captar esses gases de efeito estufa, pois consiste em um sistema fechado com uma saída que pode ser conectada a um queimador, para que ocorra a transformação de gases altamente prejudiciais em gases menos prejudiciais ao aquecimento global (UNFCCC, 2006).

Nesse contexto, cresce o número de projetos MDL (Mecanismos de Desenvolvimento Limpo) na produção suinícola com o uso de biodigestores como forma de tratamento dos efluentes. O MDL é uma forma encontrada para incentivar as reduções das emissões de gases de efeito estufa na atmosfera, em que as unidades produtoras recebem remuneração para cada tonelada de $\mathrm{CO}_{2}$ (crédito de carbono) que deixa de ser emitida ou que é retirada da atmosfera (ROCHA, 2003).

Com a venda dos créditos de carbono e a utilização do biogás como fonte de energia para a granja, o suinocultor consegue reduzir seus custos de produção. Com a redução dos custos, o produtor, muitas vezes, acaba utilizando o capital para investir apenas na ampliação de seu plantel de suínos, sem se preocupar com o sistema de tratamento dos dejetos.

A ampliação do plantel provoca aumento na quantidade de dejetos, fazendo com que diminua o tempo de permanência do material dentro do biodigestor (redução do tempo de retenção hidráulica) e com isso o substrato deixa o biodigestor antes do tempo, ou seja, com potencial poluente ainda elevado. Uma maneira prática de evidenciar esse problema é quando se observa grande quantidade de gás sendo produzida pelo material que deixa o biodigestor.

Uma alternativa que possibilita o aumento de plantel sem alteração do tamanho do sistema de tratamento seria torná-lo mais eficiente. Esse aumento da eficiência pode ser conseguido com a separação da fração sólida; sendo que, assim, o processo será otimizado (maior produção de biogáskg ${ }^{-1}$ de sólido), mais rápido (menor tempo de retenção hidráulica), além de mais econômico, pois necessita de reatores com menor tamanho para o mesmo número de animais .

$\mathrm{Na}$ literatura, são poucos os trabalhos que avaliem o potencial poluidor remanescente dos efluentes de biodigestores com diferentes TRH e ainda mais escassos são os dados que relacionam o TRH com a separação ou não da fração sólida. Esse fato justifica o estudo da separação de sólidos, dos tempos de retenção hidráulica e do potencial de produção de biogás remanescente no efluente dos biodigestores, pois esse dado permitirá a avaliação do potencial de emissão de metano para a atmosfera em cada tempo de retenção adotado.

\section{MATERIAL E MÉTODOS}

A pesquisa foi desenvolvida no Laboratório de Biodigestão Anaeróbia do Departamento de Engenharia Rural da Faculdade de Ciências Agrárias e Veterinárias, pertencentes à Universidade Estadual Paulista, Câmpus de Jaboticabal - SP.

Para a avaliação do potencial poluidor remanescente, foram utilizados, neste experimento, os efluentes de biodigestores semicontínuos abastecidos com água residuária de suinocultura com separação da fração sólida (CSFS) e sem separação da fração sólida (SSFS), e conduzidos sob diferentes tempos de retenção hidráulica (15; 22; 29 e 36 dias). Essa sequência de processos pode ser mais bem compreendida na Figura 1. 


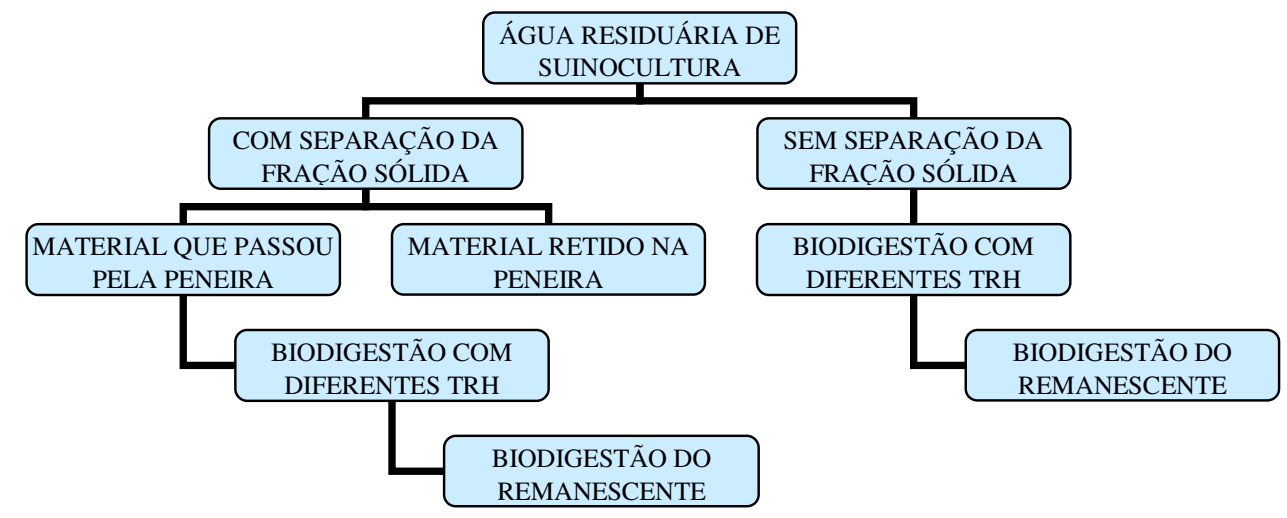

FIGURA1. Esquema da forma de obtenção de cada efluente para a avaliação do potencial poluidor remanescente. Scheme of the means of obtaining each effluent for evaluation of the remaining potential polluter.

Os efluentes dos biodigetores semicontínuos foram utilizados para o abastecimento dos biodigestores batelada de bancada, no intuito de avaliar a continuidade do processo de biodigestão anaeróbia e, consequentemente, da produção de biogás após a saída desses materiais do interior dos biodigestores semicontínuos (modelo "plug flow", comum nas suinoculturas modernas).

Durante o ensaio de biodigestão anaeróbia, foram avaliados a produção total de biogás e de metano, os potenciais de produção de biogás e de metano, demandas química e bioquímica de oxigênio (DQO e DBO) e as reduções de sólidos totais e voláteis (ST e SV).

Os biodigestores utilizados foram constituídos por vidros com capacidade de $2 \mathrm{~L}$ de substrato em fermentação, fechados por uma tampa de borracha com um tubo metálico acoplado no centro para permitir a saída do biogás produzido. Os vidros foram acoplados, por meio de mangueiras de silicone a gasômetros de PVC, permitindo o armazenamento e a medida do biogás produzido. Os gasômetros foram constituídos de dois cilindros de 250 e $300 \mathrm{~mm}$ de diâmetro e encontram-se inseridos um no interior do outro, de tal forma que o espaço existente entre a parede externa do cilindro interior e a parede interna do cilindro exterior comporta um volume de água ("selo de água"), atingindo a profundidade de $500 \mathrm{~mm}$. O cilindro de $300 \mathrm{~mm}$ de diâmetro foi fixado sobre uma placa de PVC com $25 \mathrm{~mm}$ de espessura, recebendo o cilindro de $250 \mathrm{~mm}$ de diâmetro no seu interior. O cilindro de $250 \mathrm{~mm}$ diâmetro teve uma das extremidades vedadas com um cap que recebeu o gás produzido; a outra extremidade esteve emborcada no selo de água para armazenar o gás produzido (Figura 2).

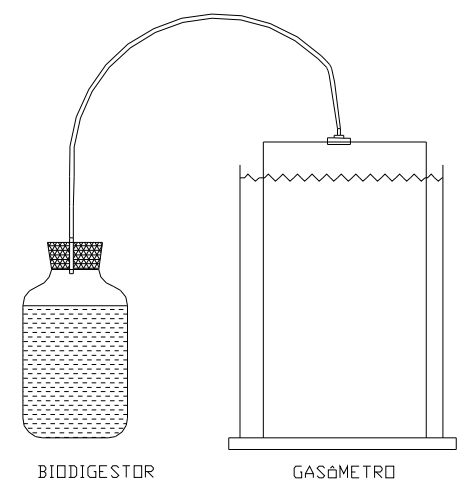

FIGURA 2. Biodigestor tipo batelada de bancada utilizado no experimento. Bench scale biodigester of batch type. 
Os teores de sólidos totais (ST), sólidos voláteis (SV), demanda química de oxigênio (DQO) e demanda bioquímica de oxigênio (DBO) das amostras coletadas durante o ensaio de biodigestão anaeróbia foram determinados de acordo com metodologia descrita por APHA (1995).

Para a determinação dos volumes de biogás produzidos diariamente, mediu-se o deslocamento vertical dos gasômetros, que foi multiplicado pela área da seção transversal interna dos gasômetros, ou seja, $0,0507 \mathrm{~m}^{2}$. Após cada leitura, os gasômetros foram zerados, utilizando-se do registro de descarga do biogás. A correção do volume de biogás para as condições de 1 atm e $20{ }^{\circ} \mathrm{C}$ foi efetuada com base no trabalho de CAETANO (1985).

Os potenciais de produção de biogás foram calculados utilizando-se dos dados de produção diária e das quantidades de substrato, de ST, de SV adicionados nos biodigestores, além das quantidades de SV reduzidos durante o processo de biodigestão anaeróbia. Os valores foram expressos em $\mathrm{m}^{3}$ de biogás por $\mathrm{kg}$ de substrato, de dejetos ou de ST e de SV.

As análises da composição do biogás produzido em biodigestores abastecidos com água residuária de suinocultura foram realizadas semanalmente para a determinação dos teores de metano $\left(\mathrm{CH}_{4}\right)$ e de dióxido de carbono $\left(\mathrm{CO}_{2}\right)$, principalmente em cromatógrafo de fase gasosa Finigan GC2001, equipado com as colunas Porapack Q e Peneira Molecular, e detector de condutividade térmica (STEIL, 2001).

O delineamento utilizado foi o inteiramente casualizado, em esquema fatorial $2 \times 4$, sendo dois tipos de substrato (efluente de biodigestores semicontínuos SSFS e CSFS), em quatro tempos de retenção hidráulica $(15 ; 22 ; 29$ e 36 dias), com três repetições por tratamento. As médias dos tratamentos foram comparadas entre si por meio do teste de Tukey, a 1\% de significância. A análise estatística foi feita por meio do SAS (1990).

\section{RESULTADOS E DISCUSSÃO}

As produções e os potenciais de produção de biogás e de metano encontradas no experimento estão apresentadas nas Tabelas 1 e 2 .

TABELA 1. Produção (litros) e potenciais de produção de biogás (litros $\mathrm{kg}^{-1}$ substrato, ST, SV, DQO e DBO adicionado). Production (liters) and potential production of biogas (liters $\mathrm{kg}^{-1}$ substrate, TS, VS, COD and BOD added).

\begin{tabular}{|c|c|c|c|c|c|c|}
\hline \multirow{2}{*}{$\mathrm{TRH}$} & \multicolumn{2}{|c|}{ Litro } & \multicolumn{2}{|c|}{ Litro $\mathrm{kg}^{-1}$ Substrato } & \multicolumn{2}{|c|}{ Litro $\mathrm{kg}^{-1} \mathrm{ST}$} \\
\hline & SSFS & CSFS & SSFS & CSFS & SSFS & CSFS \\
\hline 15 & $9,2 \mathrm{Aa}$ & $1,8 \mathrm{Ba}$ & $3,1 \mathrm{Aa}$ & $0,6 \mathrm{Ba}$ & $396,0 \mathrm{Aa}$ & $108,0 \mathrm{Ba}$ \\
\hline 22 & $4,1 \mathrm{Ab}$ & $1,0 \mathrm{Bab}$ & $1,4 \mathrm{Ab}$ & $0,3 \mathrm{Bab}$ & $212,0 \mathrm{Ab}$ & $63,0 \mathrm{Bab}$ \\
\hline 29 & $2,4 \mathrm{Ac}$ & $0,8 \mathrm{Bb}$ & $0,8 \mathrm{Ac}$ & $0,3 \mathrm{Bb}$ & $128,0 \mathrm{Ac}$ & $66,0 \mathrm{Bab}$ \\
\hline 36 & $1,4 \mathrm{Ad}$ & $0,2 \mathrm{Bb}$ & $0,5 \mathrm{Ad}$ & $0,1 \mathrm{Bb}$ & 73,0Ad & $17,0 \mathrm{Bb}$ \\
\hline C.V. \% & \multicolumn{2}{|c|}{14,39} & \multicolumn{2}{|c|}{14,38} & \multicolumn{2}{|c|}{17,33} \\
\hline \multirow{2}{*}{ TRH } & \multicolumn{2}{|c|}{ Litro $\mathrm{kg}^{-1} \mathrm{SV}$} & \multicolumn{2}{|c|}{ Litro $\mathrm{kg}^{-1} \mathrm{DQO}$} & \multicolumn{2}{|c|}{ Litro $\mathrm{kg}^{-1}$ DBO } \\
\hline & SSFS & CSFS & SSFS & CSFS & SSFS & CSFS \\
\hline 15 & $501,0 \mathrm{Aa}$ & $151,0 \mathrm{Ba}$ & $185,0 \mathrm{Aa}$ & $62,0 \mathrm{Ba}$ & $311,0 \mathrm{Aa}$ & $80,0 \mathrm{Ba}$ \\
\hline 22 & $293,0 \mathrm{Ab}$ & $88,0 \mathrm{Bab}$ & $105,0 \mathrm{Ab}$ & $34,0 \mathrm{Bb}$ & $174,0 \mathrm{Ab}$ & $44,0 \mathrm{Bb}$ \\
\hline 29 & 179,0Ac & 91,0Bab & $70,0 \mathrm{Ac}$ & $29,0 \mathrm{Bc}$ & $111,0 \mathrm{Ac}$ & $39,0 \mathrm{Bbc}$ \\
\hline 36 & $101,0 \mathrm{Ad}$ & $24,0 \mathrm{Bb}$ & $51,0 \mathrm{Ad}$ & $10,0 \mathrm{Bc}$ & $66,0 \mathrm{Ad}$ & $12,0 \mathrm{Bc}$ \\
\hline
\end{tabular}

Na linha, letras maiúsculas comparam resultados com e sem separação da fração sólida e, na coluna, letras minúsculas comparam os tempos de retenção hidráulica. Médias seguidas de letras distintas diferem entre si, pelo teste de Tukey $(\mathrm{P}<0,01)$. 
TABELA 2. Produção (litros) e potenciais de produção de metano (litros $\mathrm{kg}^{-1}$ substrato, ST, SV, DQO e DBO adicionado). Production (liters) and potential production of methane (liters $\mathrm{kg}^{-1}$ substrate, TS, VS, COD and BOD added).

\begin{tabular}{|c|c|c|c|c|c|c|}
\hline \multirow{2}{*}{ TRH } & \multicolumn{2}{|c|}{ Litro } & \multicolumn{2}{|c|}{ Litro $\mathrm{kg}^{-1}$ Substrato } & \multicolumn{2}{|c|}{ Litro $\mathrm{kg}^{-1} \mathrm{ST}$} \\
\hline & SSFS & CSFS & SSFS & CSFS & SSFS & CSFS \\
\hline 15 & $7,0 \mathrm{Aa}$ & $1,4 \mathrm{Ba}$ & $2,3 \mathrm{Aa}$ & $0,5 \mathrm{Ba}$ & $305,0 \mathrm{Aa}$ & $84,0 \mathrm{Ba}$ \\
\hline 22 & $3,1 \mathrm{Ab}$ & $0,7 \mathrm{Bb}$ & $1,0 \mathrm{Ab}$ & $0,2 \mathrm{Bb}$ & $163,0 \mathrm{Ab}$ & $48,0 \mathrm{Bab}$ \\
\hline 29 & $1,8 \mathrm{Ac}$ & $0,6 \mathrm{Bb}$ & $0,6 \mathrm{Ac}$ & $0,2 \mathrm{Bb}$ & $96,0 \mathrm{Ac}$ & $50,0 \mathrm{Bab}$ \\
\hline 36 & $1,0 \mathrm{Ad}$ & $0,2 \mathrm{Bb}$ & $0,3 \mathrm{Ad}$ & $0,1 \mathrm{Bb}$ & $53,0 \mathrm{Ad}$ & $13,0 \mathrm{Bb}$ \\
\hline C.V. \% & & & & & & \\
\hline \multirow{2}{*}{ TRH } & \multicolumn{2}{|c|}{ Litro $\mathrm{kg}^{-1} \mathrm{SV}$} & \multicolumn{2}{|c|}{ Litro $\mathrm{kg}^{-1} \mathrm{DQO}$} & \multicolumn{2}{|c|}{ Litro $\mathrm{kg}^{-1} \mathrm{DBO}$} \\
\hline & SSFS & CSFS & SSFS & CSFS & SSFS & CSFS \\
\hline 15 & $385,0 \mathrm{Aa}$ & $117,0 \mathrm{Ba}$ & $144,0 \mathrm{Aa}$ & $48,0 \mathrm{Ba}$ & $240,0 \mathrm{Aa}$ & $62,0 \mathrm{Ba}$ \\
\hline 22 & $226,0 \mathrm{Ab}$ & $68,0 \mathrm{Bab}$ & $811,0 \mathrm{Ab}$ & $26,0 \mathrm{Bb}$ & $135,0 \mathrm{Ab}$ & $34,0 \mathrm{Bb}$ \\
\hline 29 & $134,0 \mathrm{Ac}$ & $69,0 \mathrm{Bab}$ & $53,0 \mathrm{Ac}$ & $22,0 \mathrm{Bbc}$ & $83,0 \mathrm{Ac}$ & $29,0 \mathrm{Bbc}$ \\
\hline 36 & $74,0 \mathrm{Ad}$ & $18,0 \mathrm{Bb}$ & $37,0 \mathrm{Ac}$ & $7,0 \mathrm{Bc}$ & $48,0 \mathrm{Ad}$ & $10,0 \mathrm{Bc}$ \\
\hline C.V. \% & \multicolumn{2}{|c|}{16,97} & \multicolumn{2}{|c|}{14,28} & \multicolumn{2}{|c|}{11,26} \\
\hline
\end{tabular}

Na linha, letras maiúsculas comparam resultados com e sem separação da fração sólida e, na coluna, letras minúsculas comparam os tempos de retenção hidráulica. Médias seguidas de letras distintas diferem entre si, pelo teste de Tukey $(\mathrm{P}<0,01)$.

Os resultados referentes às produções e aos potenciais de produção de biogás e de metano encontrados neste trabalho foram extremamente elevados, principalmente para o efluente que não sofreu separação da fração sólida e manejado no TRH de 15 dias (Tabelas 1 e 2).

Esses valores indicam que o material SSFS necessita de maior tempo no interior dos biodigestores antes de serem lançados no meio, pois as produções de metano remanescentes foram 7,6 vezes maiores no TRH de 15 em relação ao de 36 dias. Em média, houve redução de 0,095 litro de $\mathrm{CH}_{4} \mathrm{~kg}^{-1}$ de substrato para cada dia que o material ficou a mais dentro dos biodigestores semicontínuos.

Em estudo sobre os potenciais de produção de metano dos dejetos de diversas espécies de animais, MOLLER et al. (2004) calcularam para a espécie suína um potencial médio de $516 \mathrm{~L} \mathrm{CH}_{4} \mathrm{~kg}^{-1}$ de $\mathrm{SV}$ adicionado. Comparando esse potencial com os potenciais remanescentes encontrados neste trabalho para SSFS, pode-se concluir que apenas $25,3 \%$ do potencial total foi obtido quando se utilizou o TRH de 15 dias. Esse dado é inferior ao obtido por SOUZA \& CAMPOS (2007), os quais, ao abastecerem biodigestores contínuos com dejetos de suínos, observaram que, quando o TRH foi de 15 dias, houve redução de $50 \%$ da capacidade total de produção de gás do material antes de sua saída do biodigestor.

O melhor desempenho foi para o efluente CSFS, com 36 dias de TRH, que chegou a um aproveitamento estimado de $96 \%$ do potencial de produção de metano, tendo como base o potencial encontrado por MOLLER et al. (2004).

Na procura por uma carga orgânica ideal para o TRH de 35 dias, SÁNCHEZ et al. (2001) abasteceram biodigestores com dejetos de suínos, adotando-se cargas de DQO de 3,3; 7,0; 12,0; 19,3 e $26,3 \mathrm{~g} \mathrm{O}_{2}$ L $^{-1}$. Foi observado que, com o aumento de DQO na composição dos substratos, houve menor redução de suas concentrações durante a biodigestão anaeróbia, reduzindo, assim, a eficiência do processo, o que levou os autores a concluírem que o tempo de retenção hidráulica não foi suficiente. Os autores estimaram que aumento na concentração de DQO da ordem de $6,0 \mathrm{gO}_{2} \mathrm{~L}^{-1}$ resultou em $10 \%$ a menos de eficiência na remoção de matéria orgânica.

O maior potencial de produção de biogás e de metano remanescente do efluente SSFS pode ser atribuído ao maior tamanho médio das partículas e aos maiores teores de nutrientes de difícil degradação, que não foram removidos pelo processo de peneiramento, ou seja, são efluentes que possuem taxa de degradação mais lenta, por isso necessitam de mais tempo para completa estabilização. 
As fibras vegetais de difícil degradação são apontadas como as principais responsáveis pela redução na taxa de fermentação da massa no interior dos biodigestores. RICO et al. (2006) observaram percentagens de 7,3 e 5,7; 18,5 e 14,8; 6,0 e 24,2; 4,7 e 20,9 para gordura, proteína, celulose, hemicelulose na fração líquida (separada com peneira de $1,3 \mathrm{~mm}$ de malha) e no dejeto in natura de bovinos, respectivamente. No mesmo trabalho, os autores observaram que, para um mesmo tempo TRH, os potenciais de produção de biogás foram de 0,604 e $0,3711 \mathrm{~L} \mathrm{CH}_{4} / \mathrm{g} \mathrm{SV}$ para as frações liquidas e sólidas, respectivamente, levando à conclusão de que a fração sólida necessitaria de maior tempo para a completa estabilização.

O comportamento dos potenciais de produção de biogás e de metano remanescentes dos efluentes CSFS e SSFS, em função do TRH utilizado, pode ser observado nas Figuras 3; 4; 5 e 6.
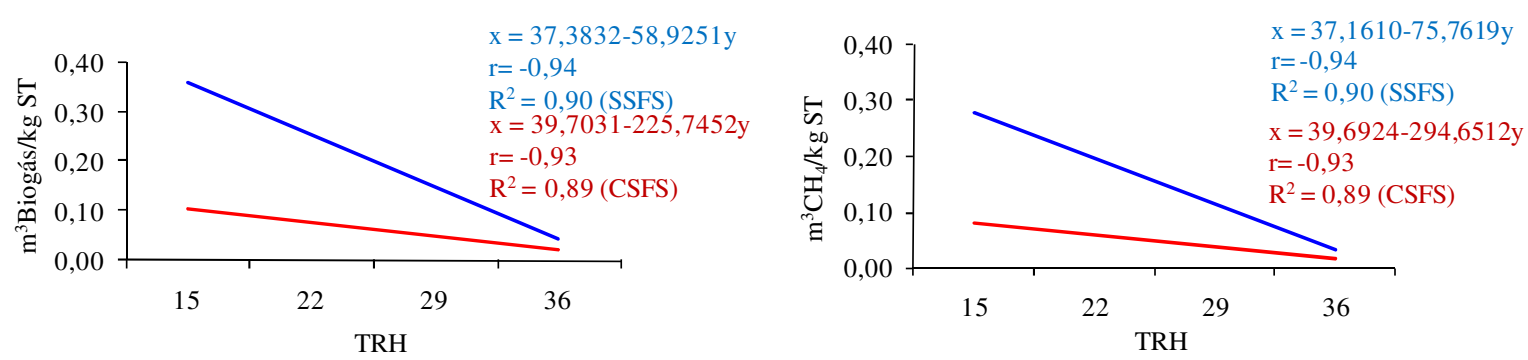

FIGURA 3. Potencial de produção de biogás e de metano remanescente por kg de ST adicionado nos substratos com e sem separação da fração sólida nos TRH de 15;22; 29 e 36 dias.

Potential production of biogas and methane per $\mathrm{kg}$ of remaining TS added in substrate with and without the separation of solid fraction in HRT 15; 22; 29 and 36 days.
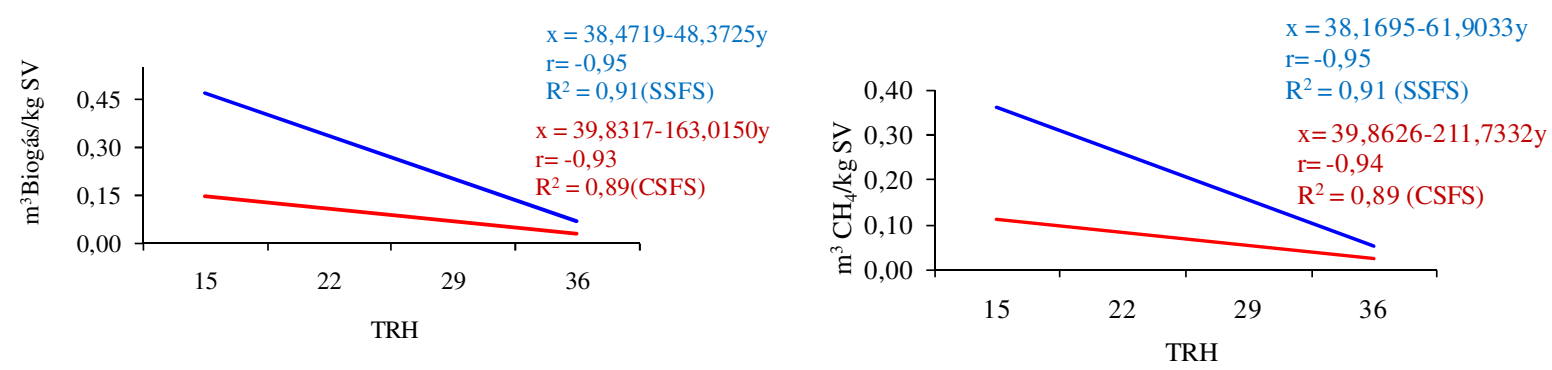

FIGURA 4. Potencial de produção de biogás e metano remanescente por kg de SV adicionado nos substratos com e sem separação da fração sólida nos TRH de 15;22; 29 e 36 dias. Potential production of biogas and methane per kg of remaining VS added in substrate with and without the separation of solid fraction in HRT 15; 22; 29 and 36 days.
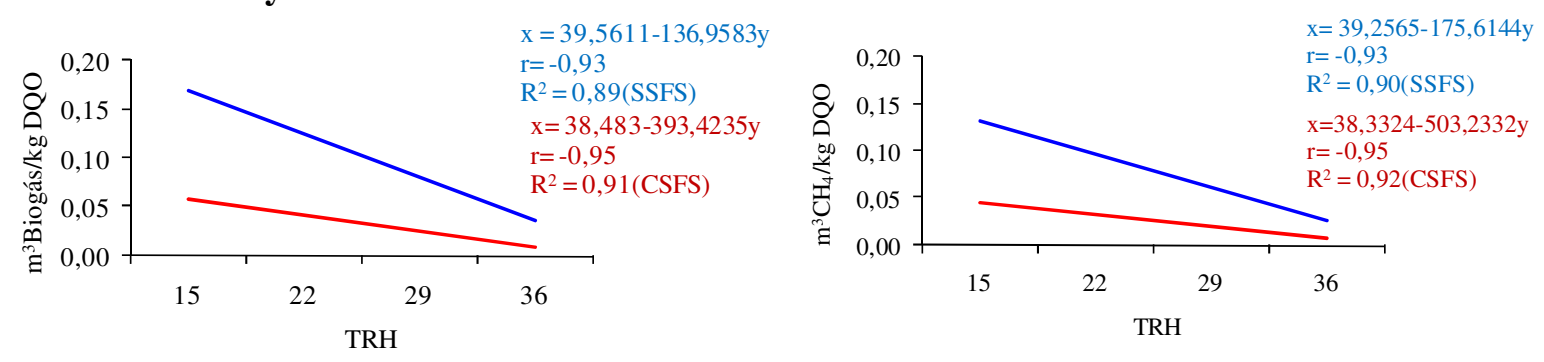

FIGURA 5. Potencial de produção de biogás e de metano remanescente por kg de DQO adicionada nos substratos com e sem separação da fração sólida nos TRH de 15; 22; 29 e 36 dias. Potential production of biogas and methane per kg of remaining in DQO added substrate with and without the separation of solid fraction in HRT 15; 22; 29 and 36 days. 

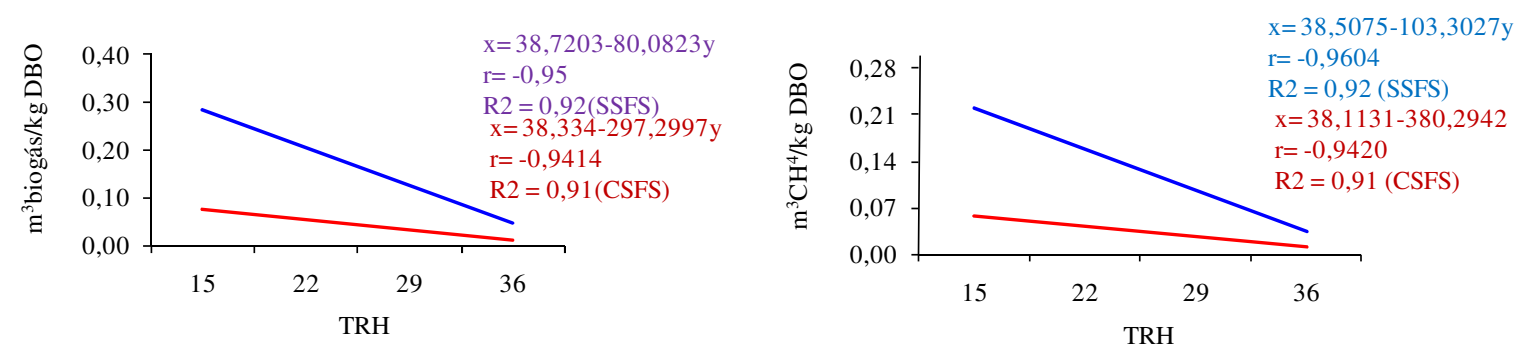

FIGURA 6. Potencial de produção de biogás e demetano remanescente por kg de DBO adicionado nos substratos com e sem separação da fração sólida nos TRH de 15;22; 29 e 36 dias. Potential production of biogas and methane per $\mathrm{kg}$ of DBO remaining in the added substrate with and without the separation of solid fraction in HRT; 15; 22; 29 and 36 days.

Como pode ser observado, tanto CSFS como SSFS apresentaram a mesma tendência, ou seja, linear com coeficiente de correlação negativo, que pode ser interpretado da seguinte forma, conforme aumentou o TRH a que o material foi submetido anteriormente, mais se reduziram os potenciais de produção biogás e de metano remanescentes. Esse comportamento já era esperado, pois os resíduos que permaneceram mais tempo dentro dos biodigestores (maiores TRH) acabariam sofrendo maior degradação, restando muito pouco para serem degradados após sua saída.

Ao comparar o potencial de produção de metano do efluente com os dados da Tabela 2 e a equação de predição da Figura 4, conclui-se que seria necessário TRH de 33 dias para que um efluente SSFS atingisse o mesmo potencial de produção de biogás remanescente por $\mathrm{kg} \mathrm{SV}$ adicionados do CSFS em TRH de 15 dias (117 litros biogáskg ${ }^{-1} \mathrm{SV}$ adicionado). Dessa forma, o uso da separação da fração sólida colaborou de maneira efetiva na redução das emissões de metano para atmosfera após a saída do material dos biodigestores.

\section{CONCLUSÕES}

A separação sólida pelo uso de peneira foi eficiente para reduzir a produção de biogás e metano remanescente dos efluentes de biodigestores semicontínuos. Dessa forma, o uso de peneiras pode ser indicado para situações em que o sistema de biodigestão se encontra subdimensionado ou em situações nas quais se pretende aumentar a eficiência do sistema. É necessário salientar que o material retido na peneira também deve sofrer tratamento adequado antes de ser aproveitado com o adubo orgânico.

\section{REFERÊNCIAS}

APHA. AMERICAN PUBLIC HEALTH ASSOCIATION. Standard methods for examination of water and wastewater. $19^{\text {th }}$ ed. Washington, $1995.1 .134 \mathrm{p}$.

CAETANO, L. Proposição de um sistema modificado para quantificação de biogás. 1985. 75 f. Dissertação (Mestrado em Energia na Agricultura) - Faculdade de Ciências Agronômicas, Universidade Estadual Paulista, Botucatu, 1985.

MOLLER, H.B.; SOMMER, S.G.; AHRING, B.K. Methane productivity of manure, straw and solid fractions of manure. Biomass and Bioenergy, Kidlington, v.26, p.485-495, 2004.

RICO, J.L.; GARCIA, H.; RICO, C.; TEJERO, I. Characterization of solid and liquid fractions of dairy manure with regard to their component distribution and methane production. Bioresource Technology, Oxford, v.98, n.3, p.971-979, 2006. 
ROCHA, M.T. Aquecimento global e o mercado de carbono: uma aplicação do modelo cert. 2003. 214 f. Tese (Doutorado em Economia Aplicada) - Escola Superior de Agricultura "Luiz de Queiroz", Universidade de São Paulo, Piracicaba, 2003.

SÁNCHEZ, E.; BORJA, R.; WEILAND, P.; TRAVIESO, L.; MARTÍN, A. Effect of substrate concentration and temperature on the anaerobic digestion of piggery waste in a tropical climate. Process Biochemistry, Shangai, v.37, n.5, p.483-489, 2001.

SAS. SAS/STAT. User's guide. Version 6. $4^{\text {th }}$ ed. Cary: Statistical Analysis System, 1990. 891 p.

SOUZA, C.F.; CAMPOS, J.A. Avaliação do tempo de retenção hidráulica, agitação e temperatura em biodigestores operando com dejetos de suínos. Revista Brasileira de Agroecologia, Porto Alegre, v.2, n.1, p.235-241, 2007.

STEIL, L. Avaliação do uso de inóculos na biodigestão anaeróbia de resíduos de aves de postura, frangos de corte e suínos. 2001.109 f. Dissertação (Mestrado em Biotecnologia) - Instituto de Química, Universidade Estadual Paulista, Araraquara, 2001.

UNFCCC. UNITED NATIONS FRAMEWORK CONVENTION ON CLIMATE CHANGE. Overview of project activity cycle. Disponível em: <http://unfccc.int/cdm>. Acesso em: 15 nov. 2006. 